\title{
A case study and anesthetic implications of combined glenn, TV replacement and cabg surgery
}

\begin{abstract}
Managing a patient with single ventricle is always a challenging situation for an anesthesiologist. Maintaining the single ventricle function and avoiding sudden changes in patient's hemodynamic are of most important key notes to be considered. But when a combination of operation perform on these patients, further attention is essential in using of drugs and techniques. Every wrong attempt might result in a catastrophic income. In present case, a rare 45 years-old male known case of Ebstein's anomaly, who was candidated for Glenn and tricuspid valve replacement (TVR) and coronary artery bypass graft (CABG) surgery, is presented. Necessary information in perioperative management of these patients are also provided. After the surgery, he was discharged from ICU in presence of suitable health status.
\end{abstract}

Volume 3 Issue 5 - 2015

\author{
Kianoush S, Mehrdad S, Roya S, Mehrzad R, \\ Anahita T, Mohammad AGM, Shahnaz S \\ Department of Imam Khomeini Medical and Research Center, \\ Tehran University of Medical Sciences, Iran
}

\begin{abstract}
Correspondence: Kianoush Saberi, Department of Imam Khomeini Medical and Research Center,Tehran University of Medical Sciences, Tehran, Iran, Email saberikn@yahoo.com
\end{abstract}

Received: May 29, 2015 | Published: December 21, 2015

Keywords: glenn surgery, coronary artery bypass surgery, cardiopulmonary bypass, cardiopulmonary

Abbreviations: TVR, tricuspid valve replacement; CABG, coronary artery bypass graft; $\mathrm{LV}$, left ventricle; $\mathrm{RV}$, right ventricle; $\mathrm{MR}$, mitral regurgitation; TR, tricuspid regurgitation

\section{Case report}

A 45 years old male patient that weight $70 \mathrm{~kg}$, has come to hospital with pre-history of orthopnea and decreased physical activities. Transthoracic echocardiography has taken; extreme form of Ebstein's anomaly with small size Left ventricle (LV) was demonstrated; left ventricle had moderate systolic dysfunction (LV Ejection Fraction=40\%). A huge RA due to very large atrialized right ventricle (RV), in which had a small function was also indicated. Thus the right ventricle had moderate to severe systolic dysfunction. Other diagnosis consists of: mild mitral regurgitation (MR), free tricuspid regurgitation (TR), multiple fenestration of anterior tricuspid leaflet not suitable for monocusp repair. In angiography experiment, 2VD (2 vessel disease) was reported.

The patient was candidated for combined Glenn and TV replacement and CABG surgery, simultaneously. In pre-operation laboratory test serum creatinine was $1.5 \mathrm{mg} / \mathrm{dl}$ and hemoglobin was $18 \mathrm{~g} / \mathrm{dl}$ and other liver functional experiment was also normal. In the operating room, two peripheral vessels were taken by angiocaths number 14 and 16. Arterial line was conducted through the left radial artery to provide invasive blood pressure monitoring.

Anesthesia induction commenced with $3 \mathrm{mg} / \mathrm{kg}$ sodium thiopental, $0.1 \mathrm{mg} / \mathrm{kg}$ midazolam, $7.5 \mu \mathrm{g} / \mathrm{kg}$ fentanyl and $0.2 \mathrm{mg} / \mathrm{kg}$ cisatracurium and maintained using $20-100 \mu \mathrm{g} / \mathrm{kg} / \mathrm{min}$ propofol, $0.25-0.5 \mu \mathrm{g} /$ $\mathrm{kg} / \mathrm{min}$ midazolam, fentanyl $0.5 \mu \mathrm{g} / \mathrm{kg} /$ hour, and $2-4 \mu \mathrm{g} / \mathrm{kg} / \mathrm{min}$ atracurium. After reaching a suitable muscle relaxation, the patient was intubate with endotracheal tube number 7.5 and right femoral vein cannulation was established. The patient's ABG was checked before induction, after that, before switching to the pump, throughout pump usage and after that; preferable therapeutic activities were done. Before the cardiopulmonary bypass, $1200 \mathrm{cc}$ of ringer solution was administered in order to provide a normal and stable hemodynamic situation. Prior to incision of surgery and before cannulation and starting bypass, Heparin was administered.
After providing requirement for surgery, Aorta, IVC and SVC cannulation at presence of acceptable ACT ( $>480$ s) the cardiopulmonary bypass was started. A surgery consist of Bidirectional Glenn, TVR (biologic) and CABG with 3 grafts was provided. Pump time was 200 minutes and cross Glenn time was 35 minutes. After termination of operation, at presence of well conditions and patient's warming to 36.5 degrees, ventilation was started and adjusted to maintain $\mathrm{PaCO} 220$ to $25 \mathrm{mmHg}$, $\mathrm{PH}$ between 7.40 and 7.50. Cardiopulmonary bypass was offed gradually. A loading dose of $50 \mu \mathrm{g} / \mathrm{kg}$ and then the infusion of milrinone with $0.5 \mu \mathrm{g} / \mathrm{kg} / \mathrm{min}$ and infusion of epinephrine with $0.1 \mu \mathrm{g} / \mathrm{kg} / \mathrm{min}$ dosage were started from commencement of rewarming and the patient was weaned from bypass.

After stabilization of the patient's hemodynamic and blood pressure, Protamine was administered. Haemostasis of surgery was provided. The chest was closed and the patient was transferred to the ICU. To control bleeding, 6 units of FFP, 4 units of platelet and 2 grams of Fibrinogen were given.

In ICU, the patient was kept to 30 degree head up position. Ventilator's parameters were adjusted to provide $\mathrm{PaCo} 2$ about 25 $\mathrm{mmHg}$. The patient was weaned gradually and extubated after 8 hours of entering to ICU. The inotropes was tapered gradually. Total drainage was $450 \mathrm{cc}$. After 36 hours in ICU with good situation he was discharged from ICU and transferred to the cardiac surgery ward.

\section{Discussion}

In an effort to reduce the volume load of the ventricle and to minimize the impact of rapid changes in ventricular geometry and diastolic function that accompany primary Fontan, a systematic staged approach has been developed for patients with univentricular hearts. ${ }^{1}$

Two options have gained with bidirectional Glenn. The SVC is divided and anastomosed to the undivided pulmonary arteries, creating a bidirectional cavopulmonary (Glenn) shunt. ${ }^{2}$

\section{In a single ventricle patient the following notes are considerable}

In order to prohibit dehydration, the NPO time of these patients 
should be minimized; besides, maintaining the appropriate function of the ventricle during induction of anesthesia, requires proper preload. ${ }^{3}$ Stress controlling can assist maintaining PVR: SVR ratio. ${ }^{4}$ Of note, a single ventricle patient at every age is very sensitive to every sudden hemodynamic change.

All standard non-invasive monitors are applied for induction. ${ }^{5}$ An intra-arterial catheter is placed for continuous monitoring following tracheal intubation. Cannulation of the central veins via the jugular or subclavian is avoided out of concern for the implications of thrombosis in those vessels.

The initial purpose of anesthesia is to maintain PVR: SVR ratio and ventricle's function. Premedication would lead to a more convenient anesthesia induction; yet, hypoxemia and hypoventilation should be avoided. If airway, HR, contractility and preload were maintained preferably, different approaches are present for inducing anesthesia. Preserving a heart rate more than 100 beats per minutes would prepare a cardiac output $(\mathrm{CO})$ maintenance.

Intravenous anesthetic drugs can be chosen, according to the operation type, duration and the patient's status for providing an anesthesia induction. Hypoventilation can cause a cardiac arrest; yet, though hyperventilation improves pulmonary circulation and oxygen saturation, it is also capable of severe diminution of blood pressure (BP), which as a result would decrease coronary perfusion and cardiac arrest might take place. If SVR decreased, alpha-agonist drugs can be utilized.

Acidemia would decrease heart contractility and should be cured, immediately. During surgery, a single ventricle patient with marginal ventricular function might need inotropic support. And these patients are really sensitive to the inhibitory effects of volatile anesthetics on contractility. ${ }^{6}$

For maintenance of anesthesia, narcotics, midazolam and small doses of volatiles can be used. Furthermore, a high dose of narcotics is usable in long surgeries and the patients with low ventricular contractility.

Pulmonary circulation is higher at the expiratory phase of Positive Pressure Ventilation (PPV); so, lower mean airway pressure and short inspiratory time during PPV would augment pulmonary circulation. ${ }^{7}$ Phosphodiesterase inhibitors increase contractility, and decrease PVR and SVR; probably, it can also improve ventricular relaxation. ${ }^{8} \mathrm{NO}$ selectively decreases PVR. ${ }^{9}$

Maintaining normothermia is a requirement for diminution sympathetic outflow and prohibiting shivering; because shivering and increased sympathetic outflow can cause failure in a patient with single ventricle. To maintain tissue oxygenation, $\mathrm{CO}$ and $\mathrm{HR}$ should be monitored cautiously and must be kept constant. The patients which own a big ventricle, are susceptible to dysrhythmia and should be diagnosed immediately to maintain $\mathrm{CO}$; further necessary therapeutic actions should be taken without hesitation.

Shortly before the termination of $\mathrm{CPB}$, the tracheal tube should be cleared of secretions and the lungs completely re-expanded, as PVR will be minimized at normal functional residual capacity (FRC). Both atelectasis and alveolar over distension increase PVR. A tidal volume designed to achieve a low-normal $\mathrm{PaCO} 2$ at a respiratory rate no greater than 20 is selected.

Positive end-expiratory pressure (PEEP) is only applied judiciously to preserve normal FRC, based upon investigations in Fontan patients demonstrating a significant reduction in cardiac index mediated by an increase in PVR at PEEP values over $6 \mathrm{mmHg}$.
When a Glenn operation is performed without $\mathrm{CPB}$, coordination between surgeon and anesthesiologist is really invaluable. When the surgeon applies a pressure on the lung, hypoxemia can occur. Avoiding hyperventilation and hypoventilation is really advised.

\section{Postoperative care following a glenn surgery}

After a Glenn surgery, vein blood of SVC would shed directly and non-pulsatile to pulmonary circulation in which it is done through pressure difference and gravity. Thus, the pressure difference between right atrium and the left one, is also effective in maintaining pulmonary circulation.

Atelectasis, increasing interstitial fluid and factors that cause pulmonary vasoconstriction, would increase PVR and inhibits shedding the suitable blood from the SVC to pulmonary circulation. ${ }^{10}$ Increased LAP which happen due to mitral stenosis or regurgitation and LV dysfunction, can also avoid the entrance of SVC blood into the pulmonary circulation. Diastolic function of LV which is determined by LVEDP, is additionally effective in a suitable venous return. Thus, in order to quantitate PVR, ventricular end-diastolic pressure, AV valve function, and obstruction at the atrial septum remnant, cardiac catheterization must be performed. ${ }^{11}$

During perioperative period atrial dysrhythmia that leads to in coordination between ventricular ejection and atrial discharging, might occur. These abrupt atrial dysrhythmia can cause atrial thrombosis, myocardial failure and even unexpected death. ${ }^{12}$

These patients have a higher baseline CVP after operation in comparison to before that. ${ }^{13}$ Unlike the patients that undergone TCPC procedure, their renal perfusion pressure does not have a significant reduction. After the surgery, functional status and $\mathrm{O} 2$ saturation of these patients will be amended and their polycythaemia will become better.

To avoid protein losing enteropathy after the surgery, high protein and low fat regimen was applied. Moreover, diuretic and steroids were administered for the same purpose ${ }^{14}$ After 5 days the patient was discharged from the hospital in the presence of a good condition and normal health status.

\section{Conclusion}

To avoid cataclysm in a single ventricle patient and provide stable hemodynamic, there are different actions to be taken. Of the most important notes, is to provide adequate preload by minimizing NPO time. Because final goal in these patient is to maintain PVR: SVR ratio, stress controlling can also be beneficial. Optimum ventilation can decrease the incidence rate of cardiac arrest. To maintain a suitable heart contractility and $\mathrm{CO}$, acidemia should be controlled. Maintaining normothemia is also of high value. After the procedure, choosing the right drug and food regimen can help diminishing protein enteropathy. By using recent managements, we have provided a successful anesthesia on the patient with ebstein's anomaly for the combined Glenn, TVR and CABG and discharged him from ICU with a good health condition. To assure the best way in managing these patients, further comprehensive studies are required.

\section{Acknowledgments}

None.

\section{Conflicts of Interset}

None. 


\section{References}

1. Leyvi G, Wasnick JD. Single-Ventricle Patient: Pathophysiology and Anesthetic Management. J Cardiothorac Vasc Anesth. 2010;24(1):121-130.

2. Chowdhury D. Pathophysiology of Congenital Heart Diseases. Annals of Cardiac Anaesthesia. 2007;10(1):19-26.

3. Boldt J. Volume therapy in cardiac surgery: does the kind of fluid matter? J Cardiothorac Vasc Anesth. 2009;13(6):752-763.

4. Chassot PG, Bettex DA. Anesthesia and Adult Congenital Heart Disease. Journal of Cardiothoracic and Vascular Anesthesia. 2004;20(3):414-437.

5. Alston RP. Anaesthesia for off-pump. Anesthesia and Intensive Care Medicine. 2009;10(9):421-423.

6. Maxwell BG, Eberhardt KJ. Anesthetic and perioperative care of high-risk adults with congenital heart disease: Managing ventricular dysfunction and minimal reserve. Progress in Pediatric Cardiology. 2004;38(1-2):17-21.

7. Al-Eyadhy A. Mechanical ventilation strategy following Glenn and Fontan surgeries: On going challenge! J Saudi Heart Assoc. 2009;21(3):153-157.
8. Cotts T, Khairy P, Opotowsky AR, et al. Clinical research priorities in adult congenital heart disease. Int J Cardiol. 2014;171(3):351-360.

9. Cai J, Su Z, Shi Z, et al. Nitric Oxide and Milrinone: Combined Effect on Pulmonary Circulation After Fontan-Type Procedure: A Prospective, Randomized Study. Ann Thorac Surg. 2008;86(3):882-888.

10. McGlothlin D, Ivascu N, Heerdt PM. Anesthesia and Pulmonary Hypertension. Prog Cardiovasc Dis. 2010;55(2):199-217.

11. Brown DW, Gauvreau K, Powell AJ, et al. Cardiac magnetic resonance versus routine cardiac catheterization before bidirectional Glenn anastomosis: Long-term follow-up of a prospective randomized trial. $J$ Thorac Cardiovasc Surg. 2013;146(5):1172-1178.

12. De Feo M, Renzulli A, Onorati F, et al. The risk of stroke following CABG: one possible strategy to reduce it? Int J Cardiol. 2005;98(2):261-266.

13. Bertolizio G, DiNardo JA, Laussen PC, et al. Evaluation of Cerebral Oxygenation and Perfusion With Conversion From an Arterial-toSystemic Shunt Circulation to the Bidirectional Glenn Circulation in Patients With Univentricular Cardiac Abnormalities. J Cardiothorac Vasc Anesth. 2015;29(1):95-100.

14. Szelkowski LA, Puri NK, Singh R, et al. Current trends in preoperative, intraoperative and postoperative care of the adult cardiac surgery patient. Curr Probl Surg. 2015;52(1):531-569. 